\title{
Cryo-ablation improves anti-tumor immunity through recovering tumor educated dendritic cells in tumor-draining lymph nodes
}

This article was published in the following Dove Press journal:

Drug Design, Development and Therapy

10 March 2015

Number of times this article has been viewed

Xiao-Zheng He $\mathrm{H}^{1,2}$
Qi-Fu Wang,
Shuai Han
Hui-Qing Wang
Yong-Yi Ye
Zhi-Yuan Zhu
Zhi,2

Shi-Zhong Zhang ${ }^{1,2}$

'Department of Neurosurgery, Zhujiang Hospital, Southern Medical University, Guangzhou, People's Republic of China; ${ }^{2}$ The National Key Clinic Specialty, The Neurosurgery Institute of Guangdong Province, Guangdong Provincial Key Laboratory on Brain Function Repair and Regeneration, Southern Medical University, Guangzhou, People's Republic of China; ${ }^{3}$ Department of General Surgery, Zhujiang Hospital, Southern Medical University, Guangzhou, People's Republic of China
Background: In addition to minimally invasive destruction of tumors, cryo-ablation of tumors to some extent modulated anti-tumor immunity. Cryo-ablated tumors in glioma mice models induced anti-tumor cellular immunologic response which increases the percentage of $\mathrm{CD}^{+}$and $\mathrm{CD}^{+} \mathrm{T}$ cells in blood as well as natural killer cells. As a crucial role in triggering anti-tumor immunity, dendritic cells (DCs) were educated by tumors to adopt a tolerance phenotype which helps the tumor escape from immune monitoring. This study aims to study whether cryo-ablation could influence the tolerogenic DCs, and influence anti-tumor immunity in tumor-draining lymph nodes (TDLNs).

Methods: Using the GL261 subcutaneous glioma mouse model, we created a tumor bearing group, cryo-ablation group, and surgery group. We analyzed alteration in phenotype and function of tolerogenic DCs, and evaluated the factors of anti-tumor immunity inhibition.

Results: DCs in TDLNs in GL261 subcutaneous glioma mouse model expressed tolerogenic phenotype. In contrast to surgery, cryo-ablation improved the quantity and quality of these tolerogenic DCs. Moreover, the DCs decreased the expression of intracellular interleukin-10 (IL-10) and extra-cellular IL-10. In vitro, DCs from the cryo-ablation group recovered their specific function and induced potent anti-tumor immunity through triggering $\mathrm{T}$ cells. In vivo, cryo-ablation showed weak anti-tumor immunity, only inhibiting the growth of rechallenged tumors. But many IL-10-low DCs, rather than IL-10-high DCs, infiltrated the tumors. More importantly, Tregs inhibited the performance of these DCs; and depletion of Tregs greatly improved anti-tumor immunity in vivo.

Conclusion: Cryo-ablation could recover function of tumor induced tolerogenic DCs in vitro; and depletion of Tregs could improve this anti-tumor effect in vivo. The Tregs/CD4 ${ }^{+} \mathrm{T}$ and Tregs/ CD25 $5^{+} \mathrm{T}$ cells in TDLNs inhibit DCs' activity and function.

Keywords: glioma, cryo-ablation, dendritic cells, tumor-draining lymph nodes, anti-tumor immunity

\section{Introduction}

Dendritic cells (DCs) are the most potent "professional" antigen presenting cells and play a crucial role in triggering anti-tumor-specific immune response. ${ }^{1}$ During tumor progression, however, anti-tumor immunity is destroyed while immune tolerance becomes dominant. Some studies have shown that tumor progression is associated with the impairment of the differentiation and maturation of myeloid cells, such as DCs.,3 Several studies have demonstrated that these tolerogenic DCs, which are recruited to tumor-draining lymph nodes (TDLNs) and tumor sites, are responsible for escaping tumor immunity through activating $\mathrm{CD} 4^{+} \mathrm{CD} 25^{+} \mathrm{Foxp} 3^{+}$Tregs. ${ }^{2,4,5}$ Tumor-educated tolerogenic DCs could eliminate anti-tumor immunity through inducing CD3 down-regulation and
Correspondence: Shi-Zhong Zhang Neurosurgery Institute of Guangdong, Zhujiang Hospital, Southern Medical University, 253 Gongye Road, 510282 Guangzhou, People's Republic of China Tel/fax +86 2061643267

Email hizhongzhangsmu@yeah.net 
apoptosis of T cells. ${ }^{6}$ Some investigators have reported that Tregs can up-regulate the expression of indoleamine-pyrrole 2,3-dioxygenase and B7-H4 on DCs, and these DCs in turn suppress T cell responses. ${ }^{7,8}$ Obviously, tumor tolerogenic DCs are the check point to inhibit tumor immunity and should be an important therapy target.

In recent years, cryo-ablation of tumors has been applied in many kinds of tumors, such as glioma. ${ }^{9-11}$ In addition to minimally invasive destruction of tumors, cryo-ablation of tumors to some extent modulated anti-tumor immunity. ${ }^{12}$ One study showed that cryo-ablation combined with $\mathrm{CpG}$ injections increased the accumulation of CD11 $\mathrm{c}^{+} \mathrm{DC}$ in the TDLNs. ${ }^{11}$ Another study demonstrated that almost $14 \%$ of DCs in TDLNs were antigen loaded after cryo-ablation of tumors, which is more effective than DC vaccines. ${ }^{13}$ Tumor destruction in situ provided a useful antigen source for the induction of anti-tumor immunity. ${ }^{14}$ Countless tumor necrosis cells released their content into the extra-cellular space, thus triggering an immune response. ${ }^{15,16}$ But it is unknown whether tolerogenic DCs in TDLNs experience any functional alteration after cryo-ablation. Meanwhile, mono-cryo-ablation induced immune responses are mostly weak and not sufficient for complete eradication ${ }^{17}$ and the weak immune response mechanism is not very clear.

In this paper, we concluded that cryo-ablation GL261 tumors could induce the alteration of tolerogenic DCs in TDLNs, and studied cryo-ablation GL261 tumors mechanism of anti-tumor immunity. We designed four groups, including tumor group, surgery group, cryo-ablation group, and control group, and compared these groups' DCs function. In the cryo-ablation group, we found tolerogenic DCs in TDLNs converted into activated subtype. More importantly, this anti-tumor ability of DCs only expressed in vitro, while in vivo it was not sufficient to reject a second tumor challenge. When we depleted Tregs in vivo, the anti-tumor ability in the cryo-ablation group was greatly enhanced, which means that this immunity is limited by DC subtype and Tregs.

\section{Methods}

\section{Cell lines and mice}

Seven to nine week old C57BL/6 mice and BALB/c mice were purchased from Laboratory Animal Center of Southern Medical University (Guangzhou, People's Republic of China). Mice were bred, housed, and treated according to the approved institutional animal protocols.

The mice glioma cell line, GL261 cell, was grown in Roswell Park Memorial Institute (RPMI) 1640 medium containing 10\% fetal calf serum (FCS) (Hyclone, Logan, UT, USA). Lymphocytes from lymph nodes (LNs) were cultured in RPMI supplemented with $10 \%$ heat-inactivated fetal calf serum, $2 \mathrm{mM}$ L-glutamine, $100 \mathrm{U} / \mathrm{mL}$ penicillin, $100 \mu \mathrm{g} / \mathrm{mL}$ streptomycin, $1 \times$ nonessential amino acids and $50 \mu \mathrm{M} \beta$-mercaptoethanol. Cells were cultured in a humidified atmosphere containing $5 \%$ carbon dioxide at $37^{\circ} \mathrm{C}$.

\section{Animal model establishment}

GL261 cells were grown in $15 \mathrm{~cm}$ plates and harvested with trypsin. After washing three times with phosphate buffered saline (PBS), $10^{6}$ GL261 cells were re-suspended in $100 \mu \mathrm{L}$ PBS. For tumor implantation, 180 mice were injected with these GL261 cells intradermally 14 days prior to day 0. Tumors were measured every 4 to 5 days using Vernier calipers with the formula: $\mathrm{A} \times \mathrm{B}$, in which $\mathrm{A}$ is the largest dimension and $\mathrm{B}$ the shortest. At day 0 the mice were treated with cryo-ablation or surgery. Two weeks later, tumors from 40 mice were selected for treatments (the DCs, TDLNs or T lymphocytes examination) when tumor sizes (the tumor diameters) were calculated to be between 5 (shortest diameter) and $9 \mathrm{~mm}$ (largest diameter) (generally 2 weeks after cryoablation or surgery). The remaining mice (120), after GL261 cells treatment, were randomized into four groups; tumor free group as a control, tumor bearing group, cryo-ablation group, and surgery group. Eighty mice were involved in rechallenge experiment. After 2 weeks treatment, a total of $10^{4}$ GL261 cells in $100 \mu \mathrm{L}$ PBS were injected into the same flank. The inflammatory infiltrates, the macrophages and DCs at the tumor site were the same in the tumor bearing group, cryoablation group, and surgery group before rechallenges.

\section{Cryo-ablation or surgery}

As described, when tumors reached 5 to $9 \mathrm{~mm}$ in diameter, 20 mice underwent cryo-ablation (cryo-ablation group), and 20 mice underwent surgery (surgery group). First the mice were anesthetized with pentobarbital sodium $(50 \mathrm{mg} / \mathrm{kg}$, intra-peritoneally) and the tumor area was shaved. After $70 \%$ alcohol rinse, surgery or cryo-ablation was performed using the cryo-ablation system (Endocare, Irvine, CA, USA). A $2 \mathrm{~mm}$ cryo-ablation probe was inserted into the center of the tumor mass prior to $30 \mathrm{~s}$ cryo-ablation at $-140^{\circ} \mathrm{C}$ by triple-cycle freezing and subsequent rewarming to $30^{\circ} \mathrm{C}$ for another 3 minutes. Cryo-ablation was considered successful when the entire tumor appeared frozen macroscopically. For surgery, tumors were cut off as much as possible.

\section{Histology and Immunofluorescence}

Rechallenged tumors were taken from the cryo-ablation group and surgery group after being fixed with $4 \%$ paraformaldehyde and snap frozen in optimal cutting medium. Twenty 
micrometer sections were cut using a cryotome and mounted on slides. Sections were washed for 2 hours with PBS, and stained with CD11c-PE, interleukin (IL)-10 (eBiosciences, San Diego, CA, USA) antibodies overnight at $4^{\circ} \mathrm{C}$ after blocking with $10 \%$ bovine serum albumin. Samples were analyzed with Olympus FV-1000 confocal microscope.

\section{Isolation of DCs from TDLNs and T lymphocytes from spleens}

According to mice anatomy atlas, we collected axillary LNs, inguinal LNs, and other TDLNs. These LNs were minced with a scalpel blade and passed through a $100 \mu \mathrm{m}$ nylon mesh. The suspension was collected, washed three times and re-suspended in PBS. Mouse DCs were isolated from LNs using CD11c magnetic beads (Miltenyi Biotec, Bergisch Gladbach, Germany) for functional experiment on day 14. $\mathrm{T}$ lymphocytes from spleen of BALB/c or $\mathrm{C} 57 \mathrm{BL} / 6$ mice were isolated using CD3 magnetic beads on day 14 .

\section{Flow cytometric analysis}

To determine the phenotype of DCs in LNs, we stained DCs with CD11c and also CD80, CD86, and major histocompatibility complex II (MHC-II). Intracellular IL-10 (eBiosciences) staining was performed according to the manufacturer's instructions. For each staining, appropriate isotype staining was used. After fixation and permeabilization, the cells were analyzed with flow cytometry (FACSAria; BD Biosciences, San Jose, CA, USA). Absolute cell numbers of DCs were calculated by multiplying the relative cell numbers with the total number of viable cells. Samples were analyzed with FACSCalibur (BD Biosciences) using CellQuest Pro software (BD Biosciences). Geometric mean levels were recorded for samples stained for surface markers and isotype control samples to generate a fold-change ratio of geometric mean surface marker/geometric mean isotype control.

\section{Mixed lymphocyte responses}

Stimulator cells were DCs from LNs as described above. Responder T cells from spleen of BALB/c mice were isolated with staining with $\mathrm{CD} 3$ magnetic beads and incubated with carboxyfluorescein diacetate succinimidyl ester (CFSE) $(5 \mu \mathrm{M})$ (eBiosciences) at $37^{\circ} \mathrm{C}$ for 10 minutes according to the manufacturer's instruction. The reaction was stopped by adding FCS and extensive washes were performed. For the mixed lymphocyte responses, CFSE-labeled responder T cells ( $10^{4}$ cells $/ 50 \mu \mathrm{L} /$ well) were cultured in a 96-well U-bottom plate adding with various numbers of DCs. After incubation for 2 days at $37^{\circ} \mathrm{C}, 5 \%$ carbon dioxide, cells were labeled with CFSE for analysis by fluorescence activated cell sorting.
Cytotoxic T-lymphocyte (CTL) assay and enzyme-linked immunosorbent assay (ELISA)

CTL assay was performed to determine the cytotoxicity of the $\mathrm{CD}^{+} \mathrm{T}$ cells from $\mathrm{C} 57 \mathrm{BL} / 6$ mice spleens. $\mathrm{CD}^{+} \mathrm{T}$ cells were briefly co-cultured with DCs from different groups or tumor lysate pulsed DCs at a ratio of 10:1 in RPMI 1640 medium supplemented with $10 \%$ FBS and $20 \mathrm{U} / \mathrm{mL}$ IL-2 in 24-well plates. The CTL assay was performed 14 days after the rechallenge (after the DCs had been isolated). After 4 days, the stimulated $\mathrm{CD}^{+} \mathrm{T}$ cells were used as effectors and the GL261 cells as targets. CTL assay was performed by using CCK-8 kit according to the manufacturer's instructions (Dojindo Laboratories, Kumamoto, Japan). In brief, various numbers of effector cells were incubated with $5 \times 10^{3}$ GL261 cells at $37^{\circ} \mathrm{C}$ for 4 hours in $200 \mu \mathrm{L}$ RPMI 1640 medium containing $10 \%$ heat-inactivated FCS in round-bottom 96-well cell culture plates and absorbance of the solution was determined at 450 nm using a spectrophotometer. Cytotoxicity was calculated according to the following formula:

$$
\begin{aligned}
& \text { Cellular cytotoxicity }(\%)=\left\{1-\left[\mathrm{A}_{450}(\text { sample })-\right.\right. \\
& \left.\left.\mathrm{A}_{450}(\text { blank })\right] /\left[\mathrm{A}_{450}(\text { control })-\mathrm{A}_{450}(\text { blank })\right]\right\} \times 100 \text {. }
\end{aligned}
$$

\section{Treg depletion}

To deplete Tregs, mice received an intraperitoneal administration of $200 \mu \mathrm{g}$ anti-CD25 (eBioscience) antibodies on day 3 before tumor rechallenge. The Tregs were isolated and analyzed 2 weeks after rechallenge of those mice. The efficiency of Treg depletion was measured by staining of splenocytes for CD4 and CD25 surface markers. Control mice received in isotype control IgG.

\section{Statistical analysis}

Statistical analysis was performed using SPSS 13.0 software. The data were analyzed by Student's $t$-test, one-way analysis of variance and covariance analysis. Survival estimates were determined using Kaplan-Meier method. All statistical tests were two-sided. A $P$-value of less than 0.05 was considered statistically significant.

\section{Results \\ Alteration in numbers and phenotype of DCs in TDLNs after cryo-ablation in glioma mice models}

Tumors have been observed to inhibit DC maturation which is considered the initiator of tumor immunity. ${ }^{18}$ Therefore, 
this study aims to test whether cryo-ablation could induce tumor immunity through influence on DCs in glioma mice models. At first, we injected GL261 glioma cells into the left flank of C57BL/6 mice 14 days prior to day 0 and performed cryo-ablation or surgery when the tumor diameters reached 5 to $9 \mathrm{~mm}$. Fourteen days after treatment, mice were killed to remove the TDLNs according to mice anatomy atlas (Figure 1A). We isolated the DCs in TDLNs from tumor bearing group, tumor free group, cryo-ablation group, and surgery group to quantify the expression of MHC class II as well as CD86 and CD80 (Figure 1B). Compared with the medium levels of CD86 and CD80 expressed in the tumor free group,
DCs isolated from the tumor bearing group expressed low levels of CD86 and CD80. Specifically, DCs from mice whom had their tumors removed 2 weeks prior did not substantially recover the expression of CD86 and CD80, remaining at a medium level. But DCs from the cryo-ablation group had high levels of restored CD86 and CD80. DCs from all groups expressed similar MHC class II molecules (Figure 1C). We also counted the absolute numbers of DCs from TDLNs. There is no significant difference in the numbers of DCs between the tumor bearing group and the surgery group. But DCs in the cryo-ablation group significantly outnumbered the other groups (Figure 1D).

A

\begin{tabular}{|c|c|c|c|}
\hline $\begin{array}{l}\text { GL261 } \\
\text { implantation }\end{array}$ & $\begin{array}{l}\text { Cryo-ablation } \\
\text { or surgery }\end{array}$ & $\begin{array}{l}\text { TDLNs } \\
\text { removed }\end{array}$ & $\begin{array}{l}\text { Tumor } \\
\text { rechallenge }\end{array}$ \\
\hline Day-14\# & Day 0 & Day 14 & Day 28 \\
\hline
\end{tabular}

B
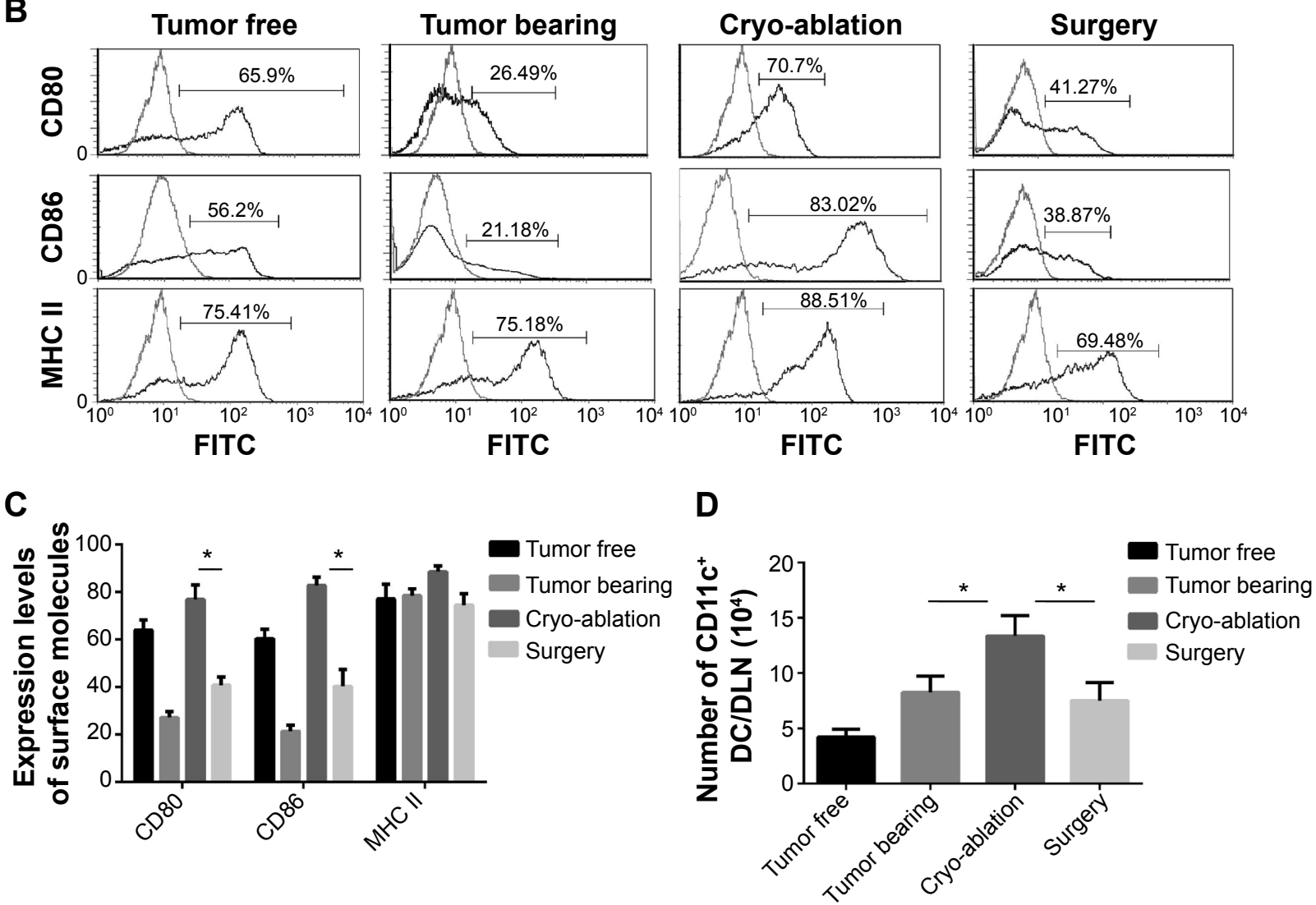

Figure I Alteration in numbers and phenotype of DCs in TDLNs after cryo-ablation in glioma mice models.

Notes: (A) Scheme to study the alteration of DCs in glioma mice models. GL26I cells $\left(10^{6}\right)$ were implanted intradermally in the left flank I4 days prior to day 0 . Two weeks later, when tumors grew to 5 to $9 \mathrm{~mm}$, mice were randomized into tumor bearing group, cryo-ablation group, and surgery group. After I4 days, TDLNs were removed and DCs were isolated. The second tumors were implanted on day 28. (B, C) Expression levels of CD80, CD86, and MHC class II in gated CDI I c DCs from four groups were evaluated by FACS analysis. Grey curves represent staining with the isotype-matched control monoclonal antibodies. (D) Absolute amounts of DCs were calculated. The data are representative of three independent experiments. Statistical significance was calculated by one-way ANOVA using the Bonferroni post-test with the following notations for $P$-value. $* P<0.05$, error bars, SEM. "Day -14 is 14 days prior to day 0.

Abbreviations: DCs, dendritic cells; TDLNs, tumor-draining lymph nodes; DLN, draining lymph node; FACS, fluorescence activated cell sorting; ANOVA, analysis of variance; SEM, standard error of the mean; MHC, major histocompatibility complex; FITC, fluorescein isothiocyanate. 


\section{Cryo-ablation converted the expression of IL- 10 in TDLN-DCs}

IL-10 was an important immunosuppressive molecule which was rarely expressed in mature DCs but was more often seen in some special DCs such as tumor induced DCs. ${ }^{6}$ Thus, we examined the IL-10 expression in DCs to evaluate the influence of cryo-ablation. DCs were identified on the gate of CD $11 \mathrm{c}^{+}$from the total TDLNs and then stained with MHC II and intracellular IL-10 (Figure 2A). Almost half of DCs from the tumor bearing group expressed intracellular IL-10, while few DCs from the tumor free group expressed intracellular IL-10. Significantly, DCs from the cryo-ablation group expressed lower intracellular IL-10 than DCs from the surgery group (Figure 2B). There was no relevance between intracellular IL-10 and MHC II. We also tested the secretion of extra-cellular IL-10 in DCs. In line with the expression of intracellular IL-10, DCs from the tumor bearing group secreted more extracellular IL-10 than DCs from the tumor free group, and DCs from the cryo-ablation group secreted less IL-10 than DCs from the surgery group (Figure 2C).

\section{The capacity of DCs to stimulate T cells enhanced after cryo-ablation}

Some reports described that tumor induced DCs had immunosuppressive functions on T cells, ${ }^{6,9}$ so we performed functional tests to examine whether cryo-ablation could promote immune
A
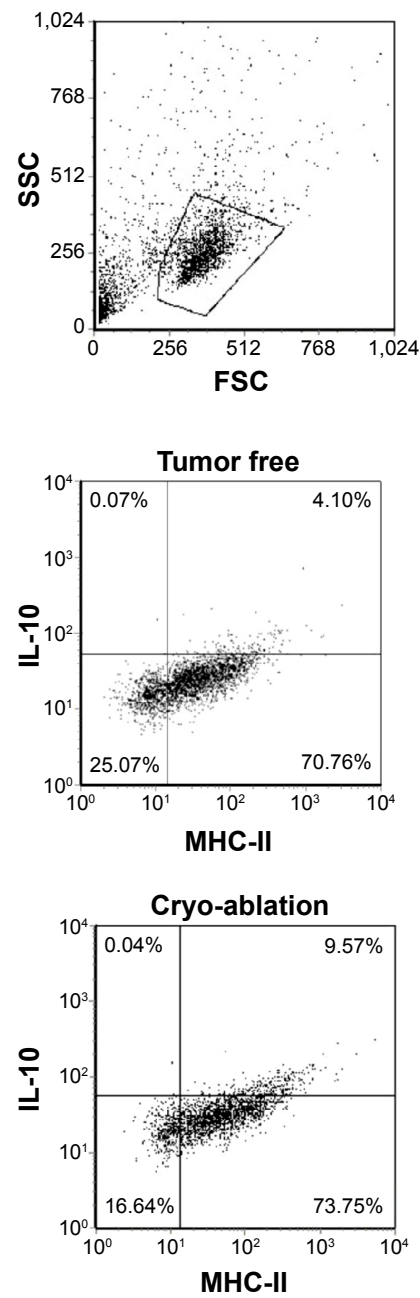
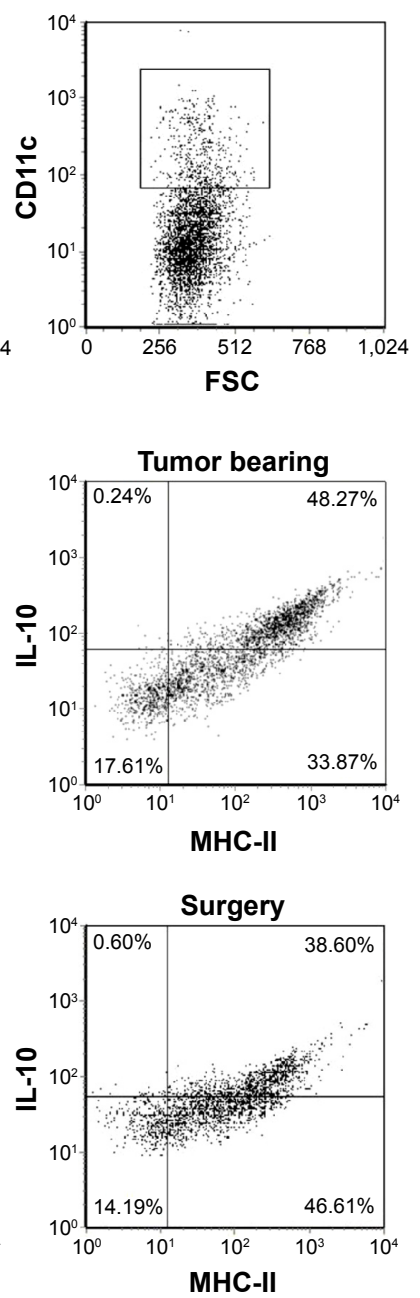

B

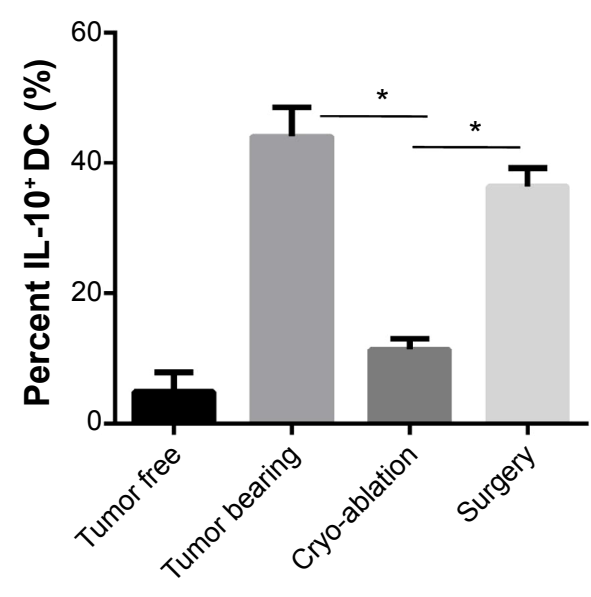

C

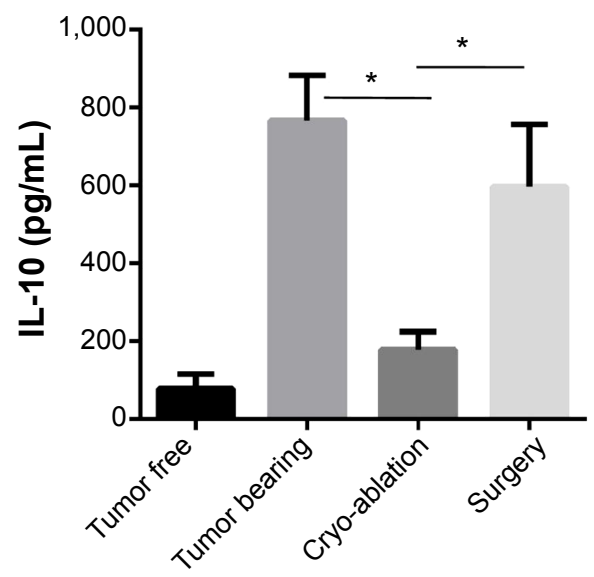

Figure 2 Cryo-ablation converted the expression of IL- I0 in TDLN-DCs.

Notes: (A) Expressions of intracellular IL-I0 in gated CDII c $\mathrm{c}^{+} \mathrm{DCs}$ from four groups were evaluated by FACS analysis. The upper left quadrant represents the IL- I0+ DCs,

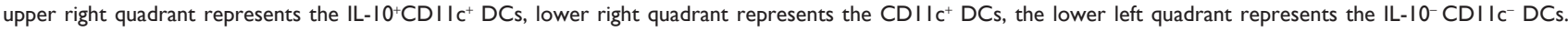
(B) Percentages of IL- I $0^{+} \mathrm{CDI} I \mathrm{c}^{+}$were calculated in the histogram. ${ }^{*} \mathrm{P}<0.05$ represents the IL- 10 levels in the cryo-ablation group compared to tumor bearing or surgery group in (C). (C) Ex vivo IL-10 secretion by $10^{4} \mathrm{DCs}$ in overnight culture, determined by ELISA, presented as the mean plus SEM of triplicate wells. The data were analyzed by Student's $t$-test. $* P<0.05$ represents the IL- $10^{+} \mathrm{DC}$ cells in the cryo-ablation group compared to tumor bearing or surgery group in Figure $2 \mathrm{~B}$.

Abbreviations: IL, interleukin; TDLN, tumor-draining lymph node; DCs, dendritic cells; FACS, fluorescence activated cell sorting; ELISA, enzyme-linked immunosorbent assay; SEM, standard error of the mean; MHC, major histocompatibility complex; FSC, forward scatter; SSC, side scatter. 
capacity of DCs in vitro. First, we tested the T cell priming capacity of DCs in an allogeneic proliferation assay. T cells isolated from BALB/c mice spleen were stained with CFSE and showed strong proliferation with anti-CD3 stimulation. Even at high DC:T cell ratio, DCs from the tumor bearing group showed weak capacity of T cell proliferation. DCs from the cryo-ablation group showed stronger $\mathrm{T}$ cell proliferation than DCs from the surgery group. The proliferation capacity was proportional with DCs:T cell ratio (Figure 3A and B). Next, we examined whether $\mathrm{T}$ cells could induce powerful cytotoxicity to GL261 cells through CTL assay. Only T cells stimulated with DCs from the cryo-ablation group induced significant cytotoxicity to GL261, while T cells stimulated with DCs from the surgery group could not induce powerful cytotoxicity to G1261 cells even at high E:T ratio (Figure 3C). When we loaded these DCs with GL261 lysates, $\mathrm{T}$ cells which were stimulated with DCs from the tumor free group showed higher cytotoxicity than those stimulated with DCs from the cryo-ablation group. But contrary to unloading
DCs, T cells which were stimulated with DCs loaded with GL261 lysates did not enhance cytotoxicity of GL261 cells (Figure 3D). Actually, for every cycle of division, a new peak with less intensity of fluorescence appeared, however, we only illustrated this in a histogram (the other cycle of division was not illustrated here). Actually Figure 1A could reflect the capacity of DCs to stimulate T cells enhanced after cryo-ablation. Additionally, Figure $3 \mathrm{~B}$ and $\mathrm{C}$ would be helpful to observe the capacity of DCs stimulating the cytotoxicity of T cells.

\section{Cryo-ablation could delay tumor growth} in tumor rechallenge and reduce the expression of IL-I0 in tumor-infiltrating DCs

To examine the anti-tumor immune memory, we re-injected GL261 glioma cells into the left flank of mice. As shown in Figure 4A, tumor growth in the surgery group was the same as the control group $(P>0.05)$, but in the cryo-ablation group, tumor grew (tumor size) significantly slower compared to the other groups $(P<0.05)$. However, the Kaplan-Meier curve
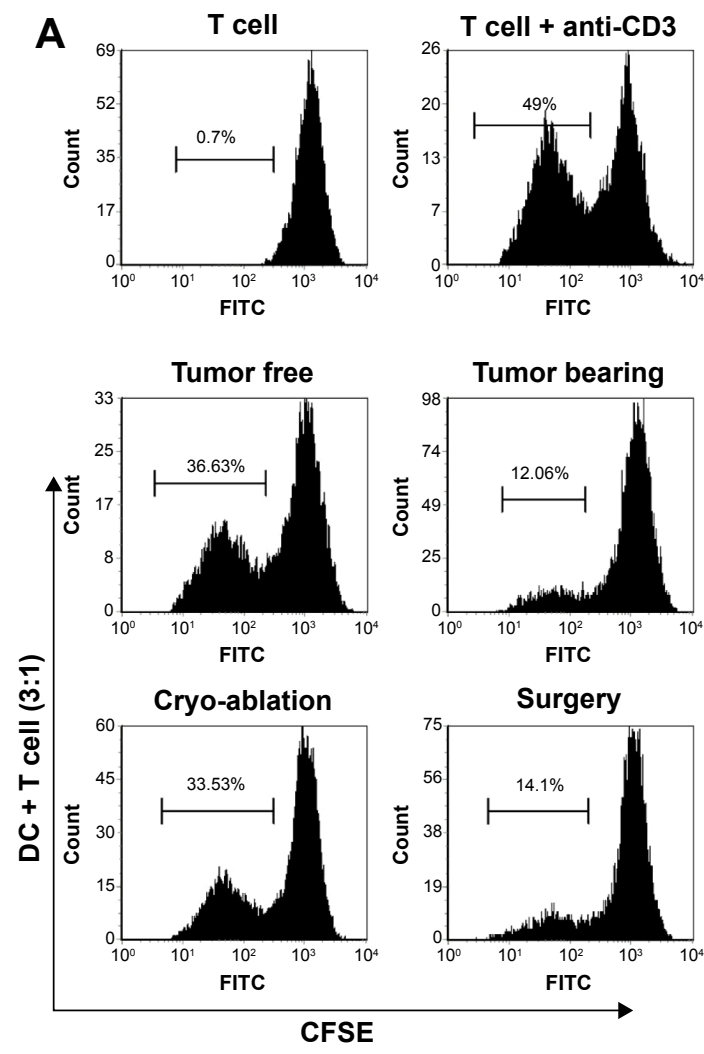

B

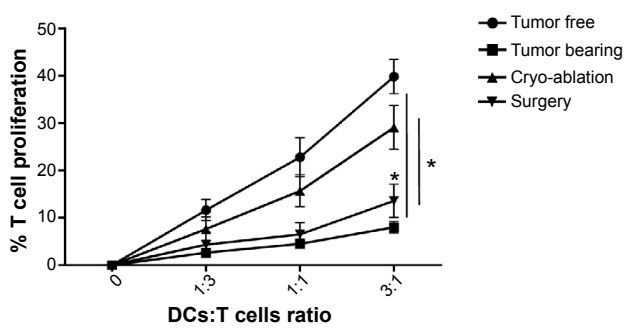

C

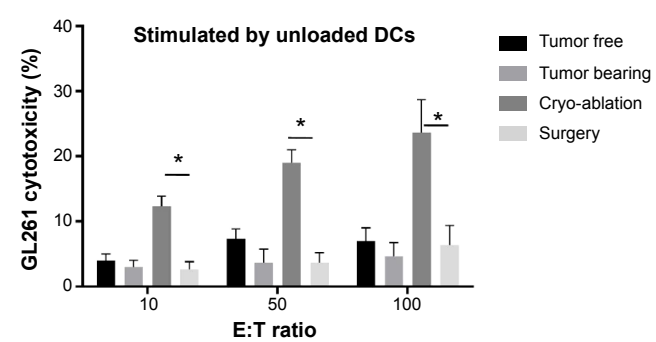

D

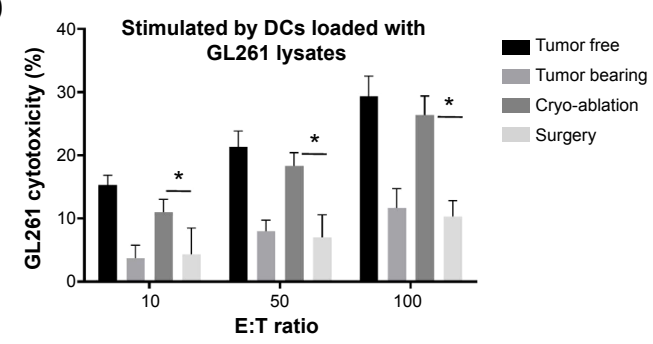

Figure 3 The capacity of DCs to stimulate T cells enhanced after cryo-ablation.

Notes: (A) Mixed lymphocyte responses were performed. Responder CD3 ${ }^{+} T$ cells were from BALB/c mice, labeled with CFSE, and incubated with the different DCs. Following incubation, the proliferation levels of cells were analyzed by FACS. (B) T cells were proliferated at various DCs:T cells ratios. (C) T cells stimulated with different DCs from four groups showed cytotoxicity to GL26I cells at various E:T ratios. (D) T cells stimulated with these DCs plus GL26I lysates showed cytotoxicity to GL26I cells at various E:T ratios. The data are representative of three independent experiments. Statistical significance was calculated by one-way ANOVA using the Bonferroni post-test with the following notations for $P$-value. $* P<0.05$, error bars, SEM.

Abbreviations: DCs, dendritic cells; CFSE, carboxyfluorescein diacetate succinimidyl ester; FACS, fluorescence activated cell sorting; ANOVA, analysis of variance; SEM, standard error of the mean; FITC, fluorescein isothiocyanate. 
showed no significant difference in the tumor-free survival time of these three groups (Figure 4B). Immunofluorescence staining showed that rechallenged tumors were infiltrated with DCs, which expressed low IL-10 in cryo-ablated mice, but high IL-10 in surgery mice (Figure 4C).

\section{Depletion of Tregs improved the survival rate of cryo-ablated mice}

DCs from cryo-ablated mice recovered normal function in vitro but showed weak anti-tumor immunity in vivo with poor survival rate in tumor rechallenge. Some papers previously showed that Tregs could inhibit DCs' activity and function, ${ }^{19,20}$ so we tested whether Tregs could impair the survival rate of cryo-ablated mice. Tregs stained with CD25 and CD4 were examined by FACS as shown in Figure 5A. The Tregs/CD4 ${ }^{+} \mathrm{T}$ ratios in tumor bearing, cryo-ablation, and surgery groups were all higher than the Tregs $/ \mathrm{CD}^{+} \mathrm{T}$ ratios in the tumor free group. There was no difference between the cryo-ablation group and surgery group. To deplete Tregs, we administered CD25-depleting antibodies intra-peritoneally and the depletion rate was above 95\% (Figure 5A). Twenty mice were involved to examine the survival by using KaplanMeier curve analysis. The Kaplan-Meier curve showed the tumor-free survival time of the rechallenge groups upon the depletion of Tregs (Figure 5B). Depletion of Tregs did not extend the survival time of the tumor bearing group and surgery group. Apparently, depletion of Tregs significantly prolonged the survival time of the cryo-ablation group and increased the long-term survival rate. The above results indicated that Tregs $/ \mathrm{CD} 4^{+} \mathrm{T}$ or Tregs $/ \mathrm{CD} 25^{+} \mathrm{T}$ cells in TDLNs inhibit the DCs' activity and function.

\section{Discussion}

In our study, we successfully isolated DCs of TDLNs from four groups, including tumor bearing group, cryo-ablation group, surgery group, and control group (tumor free group), and analyzed these different DCs' phenotype and function. These DCs from the cryo-ablation group showed effective anti-tumor immunity in vitro but weak immunity in vivo. Depletion of Tregs significantly enhanced the survival rate in

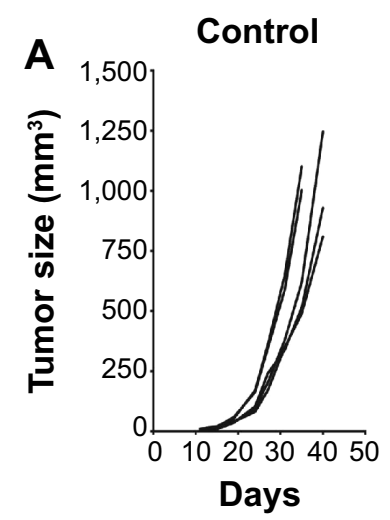

B
Surgery

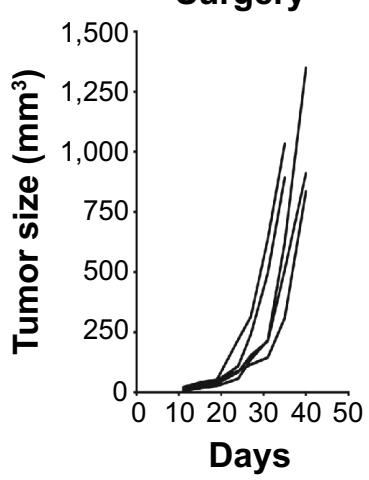

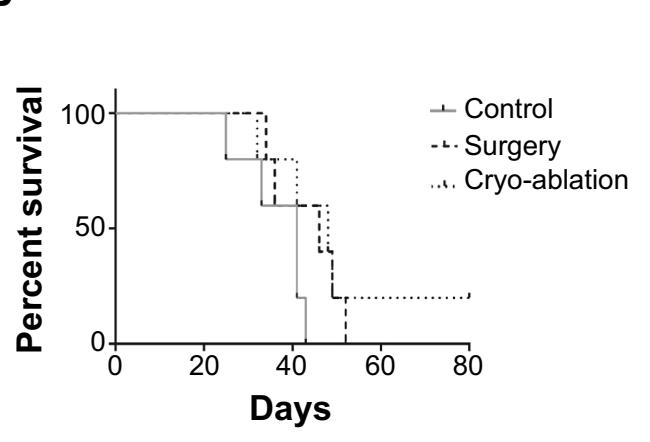

Cryo-ablation
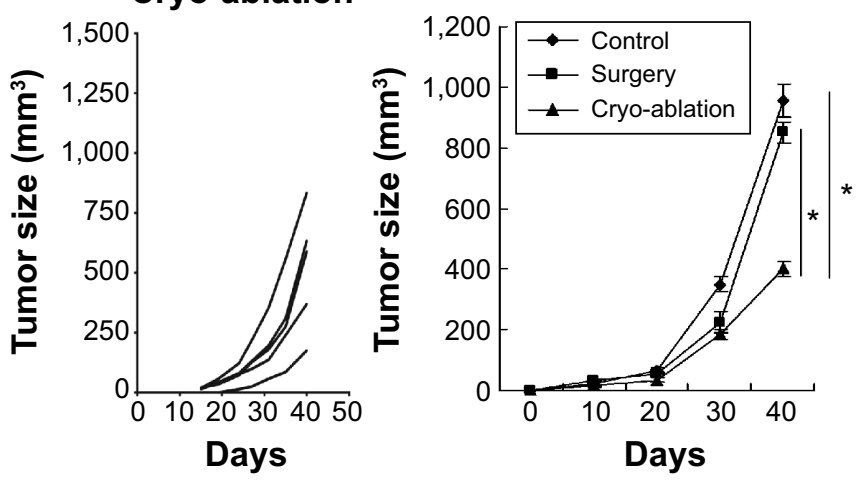

C

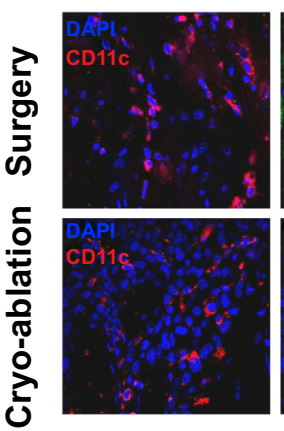

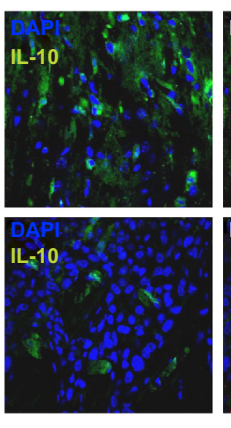

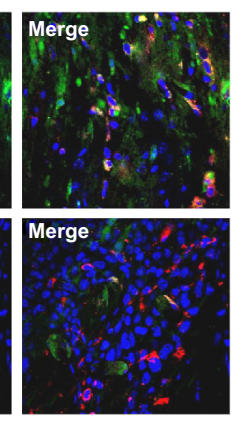

Figure 4 Cryo-ablation could delay tumor growth in tumor rechallenge and reduce the expression of IL-I0 in tumor-infiltrating DCs.

Notes: (A) Mice were re-injected $10^{4} \mathrm{GL} 26 \mathrm{I}$ cells in the left flank. Tumors were measured every 4 to 5 days using Vernier calipers. Data are pooled from five mice/group. The tumor size in each group was also analyzed and compared between groups. (B) Second tumor-free survival was plotted as a Kaplan-Meier curve and the log rank test was used for statistical analysis. However, no significant difference was discovered in the tumor-free survival time of these three groups. (C) Immunofluorescence microscopy of $20 \mu \mathrm{m}$ thick sections of second tumors from surgery group (top) and cryo-ablation group (bottom) were stained for CDI Ic (red) and IL-I0 (green). DAPI was blue. Scale bar: $20 \mu \mathrm{m}$. The data are representative of three independent experiments. Statistical significance was calculated by one-way ANOVA using the Bonferroni post-test with the following notations for $P$-value. $* P<0.05$, error bars, SEM.

Abbreviations: IL, interleukin; DCs, dendritic cells; DAPI, 4',6-diamidino-2-phenylindole; ANOVA, analysis of variance; SEM, standard error of the mean. 
A
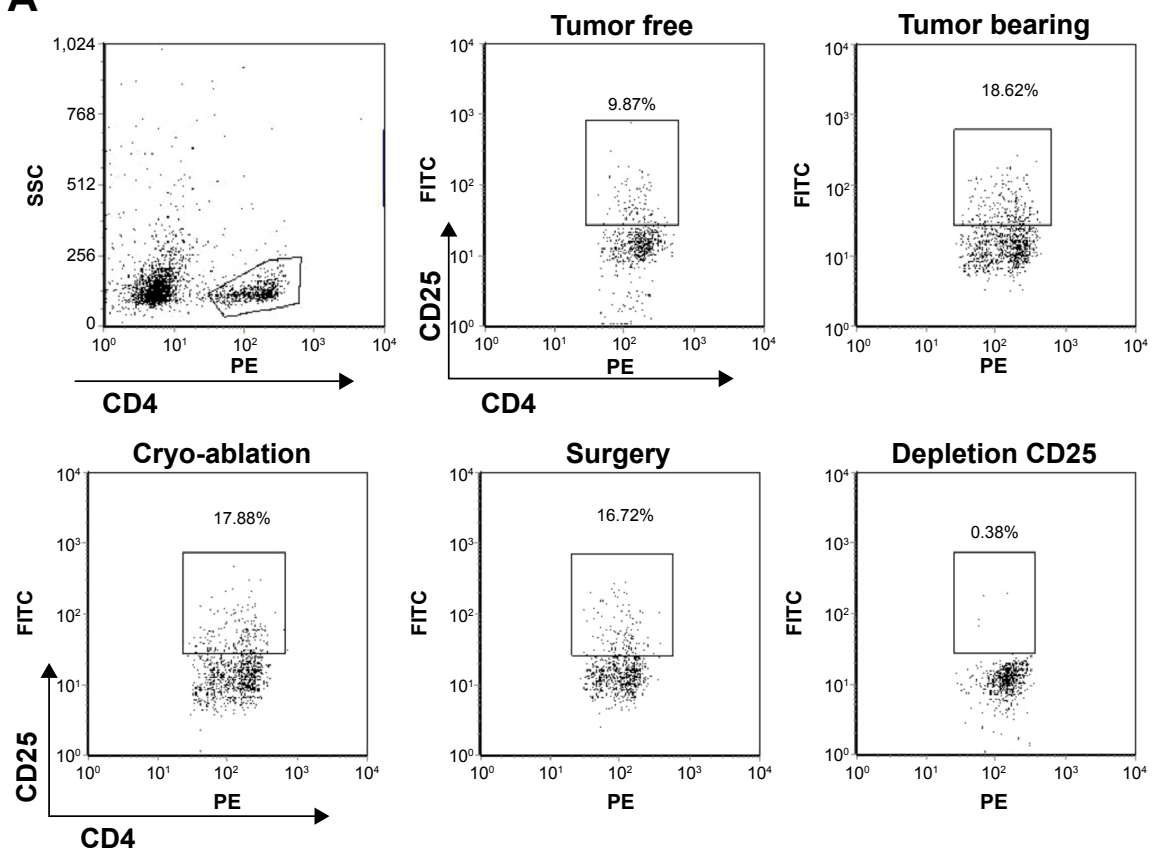

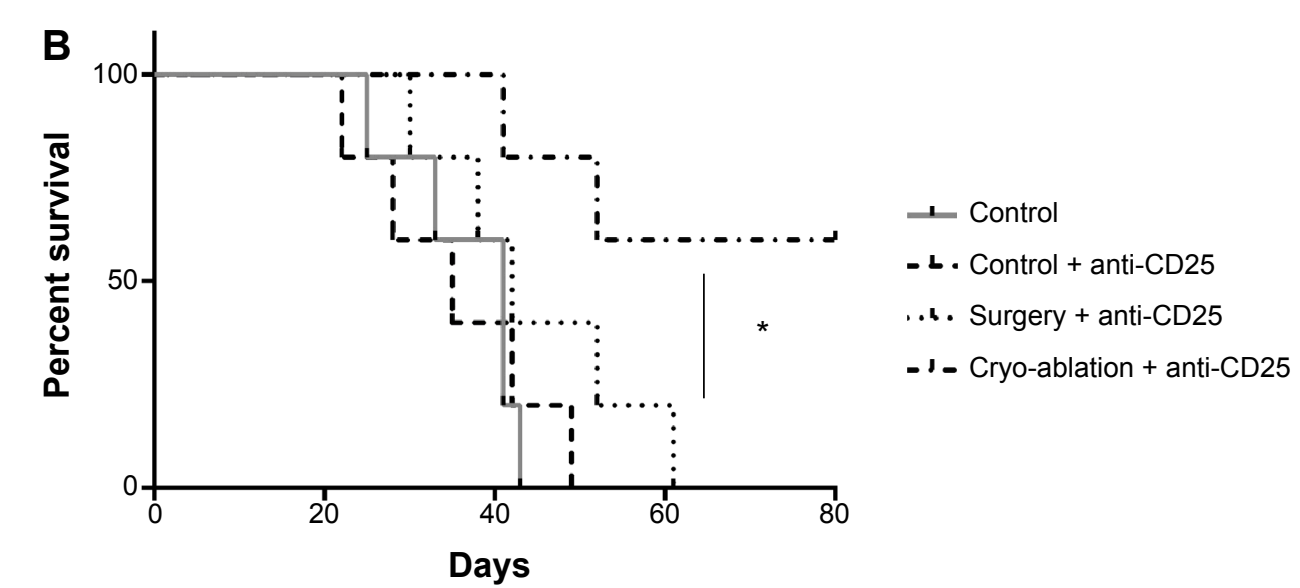

Figure 5 Depletion of Tregs improves the survival rate of cryo-ablated mice.

Notes: (A) Percentages of CD25+CD4+Tregs in CD4 ${ }^{+} \mathrm{T}$ cells from groups were shown by FACS. (B) Depletion of $C D 25^{+} \mathrm{CD} 4^{+} \mathrm{Tregs}$ improves anti-tumor effect in cryoablation group. Kaplan-Meier curve showing tumor-free survival of mice with surgery, cryo-ablation, and no treatment with depletion of $\mathrm{CD}^{2} 5^{+} \mathrm{CD} 4^{+} \mathrm{Tregs}$. Data were pooled from three independent experiments. The log rank test was used for statistical analysis. $* P<0.05$ represents survival time of the cryo-ablation and anti-CD25 group compared to the other three groups in $\mathbf{B}$.

Abbreviations: FACS, fluorescence activated cell sorting; FITC, fluorescein isothiocyanate; PE, phycoerythrin; SSC, side scatter.

the second tumor challenge, suggesting that Tregs impaired DCs' function in the cryo-ablation group.

IL-10 is a well-known immuno-modulatory cytokine which hinders anti-tumor immunity and promotes tumor growth. ${ }^{21}$ In tumor micro-environment, DCs that expressed a high level of IL-10 showed apoptosis of T cells which induced the tumor escape. ${ }^{6}$ Our preliminary experiment proved that the optimal timing of DCs isolation is 14 days, therefore, we isolated the DCs from TDLNs and spleens on day 14 (this result is not shown). In our experiment, these tolerogenic DCs were also found in TDLNs and expressed high levels of both intracellular IL-10 and extracellular IL-10. These IL-10-high DCs showed low level of co-stimulatory molecules and weak ability of $\mathrm{T}$ cell proliferation. This may be partially attributed to the fact that IL-10 has the ability of blocking DC differentiation through activation of STAT-3 and inactivation of the nuclear factor-kappa B (NF- $\kappa \mathrm{B})$ signaling pathway. ${ }^{22,23}$ A recent study showed that gliomas can up-regulate B7-H1 expression in tumor-infiltrating macrophages through modulation of autocrine/paracrine IL-10 signaling pathway, resulting in an immunosuppressive phenotype. ${ }^{24}$ Based on circumstantial evidence, in our experiment, we hypothesized that autocrine/paracrine IL-10 may also modulate 
the phenotype of DCs in TDLNs. Its mechanism will be studied in our future experiments.

In recent years, cryo-ablation of pathological tissues has become an increasingly popular method for treating tumors, including prostate, kidney, liver, bone, lung, and even glioma. Cryo-ablation is a minimally invasive surgical technique, established as rapid freezing, slow thawing, repetition of the freezing/thawing cycles. Actually, the use of cryo-ablation on glioma has no unique effects in comparison to using cryo-ablation on other tumors. In our previous study, cryo-ablation of glioma in rat models increased the percentage of $\mathrm{CD}^{+}$and $\mathrm{CD}^{+} \mathrm{T}$ cells in blood as well as the CD4/ CD8 ratio in blood. ${ }^{25}$ This study further demonstrated that cryo-ablation increased the number of DCs in TDLNs and, more importantly, inhibited the impairment of DCs caused by tumors, as well as enhancing the ability of anti-tumor immunity through $\mathrm{T}$ cells. Cryo-ablation froze most tumors and induced tumor cells' necrosis and apoptosis. These large numbers of necrotic cells which could release their cellular content (antigens, heat shock protein, and high mobility group box-1 protein) serve as immunostimulators. ${ }^{17}$ Some recent reports also showed that apoptotic cells exhibited significant immuno-stimulatory capacity. ${ }^{15,26}$ These effects of tumor cryo-ablation together re-activated tolerogenic DCs, which may be partially due to the decreased expression of IL-10. As shown in Figure 3, in the cryo-ablation group, compared with unloaded DCs, DCs loaded with GL261 lysates did not enhance cytotoxicity of T cells to GL261 (no differences between loaded or unloaded DCs from cryo-ablated mice). This result implied that the cryo-ablated tumor had released enough tumor antigens for DCs, which could not stimulate the additional cytotoxicity of $\mathrm{T}$ cells.

We also found an interesting phenomenon: the potent antitumor ability of DCs in the cryo-ablation group emerged in the in vitro experiment, but in the in vivo rechallenge experiment, these DCs displayed no efficient anti-tumor ability. Although the rechallenged tumor grew slower in the cryo-ablation group than in the surgery group, there was no significant difference in the long-term survival rate between these groups. Upon staining the rechallenged tumor, we found that the tumors of the surgery group were infiltrated by IL-10-high DCs, while those of the cyro-ablation group were infiltrated by IL-10-low DCs. That may have partially caused the slow growth of the rechallenged tumor in the cryo-ablation group.

Regulatory $\mathrm{T}$ cells are considered as the key players in tumor immunology. ${ }^{27,28}$ They accumulate in murine and human gliomas during tumor growth. ${ }^{29}$ In our subcutaneous models, the number of Tregs in TDLNs increased and almost accounted for $20 \%$ of $\mathrm{CD}^{+} \mathrm{T}$ cells. Many studies have shown that Treg depletion with anti-CD25 antibodies induced anti-tumor effect, ${ }^{13,30,31}$ however, there are some other molecules on the surface of Tregs, including CD3, CD4, etc. However, our preliminary results indicated that the other molecules (anti-CD3 or anti-CD4) showed a lower Treg depleting activity. Therefore, we used the anti-CD25 to deplete the Tregs in this study. We hypothesized that the accumulation of Tregs created an immunosuppressive micro-environment in which the recovery of DCs' function in vivo was limited. While the survival time of the other groups remained unchanged, the depletion of Tregs did significantly improve the survival time of the cryo-ablation group, which indirectly suggested that the recovery of DCs' function in vivo was inhibited by Tregs.

In conclusion, based on our previous research, we further showed that cryo-ablation could improve the anti-tumor effect through the recovery of tolerogenic DCs' function in vitro. The recovery of function was possibly associated with the expression of intracellular IL-10 and extra-cellular IL-10. The depletion of Tregs in the cryo-ablation group improves the anti-tumor effect in vivo.

\section{Acknowledgment}

This study was funded by the National Natural Science Foundation of China (Nos 81172416/H1618, 81371397/H0912).

\section{Disclosure}

The authors have no conflicts of interest to declare.

\section{References}

1. Banchereau J, Briere F, Caux C, et al. Immunobiology of dendritic cells. Annu Rev Immunol. 2000;18:767-811.

2. Sinha P, Clements VK, Bunt SK, Albelda SM, Ostrand-Rosenberg S. Cross-talk between myeloid-derived suppressor cells and macrophages subverts tumor immunity toward a type 2 response. J Immunol. 2007; 179(2):977-983.

3. Nagaraj S, Gupta K, Pisarev V, et al. Altered recognition of antigen is a mechanism of $\mathrm{CD}^{+} \mathrm{T}$ cell tolerance in cancer. Nat Med. 2007;13(7): $828-835$.

4. Ghiringhelli F, Puig PE, Roux S, et al. Tumor cells convert immature myeloid dendritic cells into TGF-beta-secreting cells inducing $\mathrm{CD} 4{ }^{+} \mathrm{CD} 25^{+}$ regulatory T cell proliferation. J Exp Med. 2005;202(7):919-929.

5. Sharma MD, Baban B, Chandler P, et al. Plasmacytoid dendritic cells from mouse tumor-draining lymph nodes directly activate mature Tregs via indoleamine 2,3-dioxygenase. J Clin Invest. 2007;117(9):2570-2582.

6. Kuang DM, Zhao Q, Xu J, Yun JP, Wu C, Zheng L. Tumor-educated tolerogenic dendritic cells induce CD3epsilon down-regulation and apoptosis of T cells through oxygen-dependent pathways. J Immunol. 2008;181(5):3089-3098.

7. Kryczek I, Wei S, Zou L, et al. Cutting edge: induction of B7-H4 on APCs through IL-10: novel suppressive mode for regulatory $\mathrm{T}$ cells. J Immunol. 2006;177(1):40-44.

8. Mellor AL, Munn DH. IDO expression by dendritic cells: tolerance and tryptophan catabolism. Nat Rev Immunol. 2004;4(10):762-774. 
9. Waitz R, Solomon SB, Petre EN, et al. Potent induction of tumor immunity by combining tumor cryo-ablation with anti-CTLA-4 therapy. Cancer Res. 2012;72(2):430-439.

10. Braun K, Wiessler M, Ehemann V, et al. Treatment of glioblastoma multiforme cells with temozolomide-BioShuttle ligated by the inverse DielsAlder ligation chemistry. Drug Des Devel Ther. 2009;2:289-301.

11. Nierkens S, den Brok MH, Roelofsen T, et al. Route of administration of the TLR9 agonist CpG critically determines the efficacy of cancer immunotherapy in mice. PLoS One. 2009;4(12):e8368.

12. Haen SP, Pereira PL, Salih HR, Rammensee HG, Gouttefangeas C. More than just tumor destruction: immunomodulation by thermal ablation of cancer. Clin Dev Immunol. 2011;2011:160250.

13. den Brok MH, Sutmuller RP, Nierkens S, et al. Efficient loading of dendritic cells following cryo and radiofrequency ablation in combination with immune modulation induces anti-tumour immunity. $\mathrm{Br} \mathrm{J}$ Cancer. 2006;95(7):896-905.

14. den Brok MH, Sutmuller RP, van der Voort R, et al. In situ tumor ablation creates an antigen source for the generation of antitumor immunity. Cancer Res. 2004;64(11):4024-4029.

15. Gallucci S, Lolkema M, Matzinger P. Natural adjuvants: endogenous activators of dendritic cells. Nat Med. 1999;5(11):1249-1255.

16. Shi Y, Rock KL. Cell death releases endogenous adjuvants that selectively enhance immune surveillance of particulate antigens. Eur $J$ Immunol. 2002;32(1):155-162.

17. Sabel MS. Cryo-immunology: A review of the literature and proposed mechanisms for stimulatory versus suppressive immune responses. Cryobiology. 2009;58(1):1-11.

18. Chaux P, Favre N, Martin M, Martin F. Tumor-infiltrating dendritic cells are defective in their antigen-presenting function and inducible B7 expression in rats. International journal of cancer. Int J Cancer. 1997;72(4):619-624.

19. Chattopadhyay G, Shevach EM. Antigen-specific induced T regulatory cells impair dendritic cell function via an IL-10/MARCH1-dependent mechanism. J Immunol. 2013;191(12):5875-5884.

20. Vignali DA, Collison LW, Workman CJ. How regulatory T cells work. Nat Rev Immunol. 2008;8(7):523-532.
21. Acuner-Ozbabacan ES, Engin BH, Guven-Maiorov E, et al. The structural network of Interleukin-10 and its implications in inflammation and cancer. BMC Genomics. 2014;15 Suppl 4:S2.

22. Gabrilovich D. Mechanisms and functional significance of tumour-induced dendritic-cell defects. Nat Rev Immunol. 2004;4(12):941-952.

23. Wang T, Niu G, Kortylewski M, et al. Regulation of the innate and adaptive immune responses by Stat-3 signaling in tumor cells. Nat Med. 2004;10(1):48-54.

24. Bloch O, Crane CA, Kaur R, Safaee M, Rutkowski MJ, Parsa AT. Gliomas promote immunosuppression through induction of B7-H1 expression in tumor-associated macrophages. Clin Cancer Res. 2013; 19(12):3165-3175.

25. Li M, Liu J, Zhang SZ, et al. Cellular immunologic response to primary cryo-ablation of C6 gliomas in rats. Technol Cancer Res Treat. 2011; 10(1):95-100.

26. Zitvogel L, Kepp O, Senovilla L, Menger L, Chaput N, Kroemer G. Immunogenic tumor cell death for optimal anticancer therapy: the calreticulin exposure pathway. Clin Cancer Res. 2010;16(12):3100-3104.

27. Curiel TJ. Tregs and rethinking cancer immunotherapy. J Clin Invest. 2007;117(5):1167-1174.

28. Wei WZ, Morris GP, Kong YC. Anti-tumor immunity and autoimmunity: a balancing act of regulatory T cells. Cancer Immunol Immunother. 2004;53(2):73-78.

29. 30. Grauer OM, Nierkens $\mathrm{S}$, Bennink E, et al. $\mathrm{CD} 4^{+} \mathrm{FoxP} 3^{+}$regulatory $\mathrm{T}$ cells gradually accumulate in gliomas during tumor growth and efficiently suppress antiglioma immune responses in vivo. Int $J$ Cancer. 2007;121(1):95-105.

30. Zarogoulidis K, Ziogas E, Boutsikou E, et al. Immunomodifiers in combination with conventional chemotherapy in small cell lung cancer: a phase II, randomized study. Drug Des Devel Ther. 2013;7: 611-617.

31. Maes W, Rosas GG, Verbinnen B, et al. DC vaccination with anti-CD25 treatment leads to long-term immunity against experimental glioma. Neuro Oncol. 2009;11(5):529-542.
Drug Design, Development and Therapy

\section{Publish your work in this journal}

Drug Design, Development and Therapy is an international, peerreviewed open-access journal that spans the spectrum of drug design and development through to clinical applications. Clinical outcomes, patient safety, and programs for the development and effective, safe, and sustained use of medicines are a feature of the journal, which

\section{Dovepress}

has also been accepted for indexing on PubMed Central. The manuscript management system is completely online and includes a very quick and fair peer-review system, which is all easy to use. Visit http://www.dovepress.com/testimonials.php to read real quotes from published authors. 